\title{
Atypical Presentation of Skull Metastasis from Rectal Adenocarcinoma as an Initial Symptom of Recurrence
}

\author{
Cemal Furat, ${ }^{1,2}$ Ahmet Hamdi Aytekin, ${ }^{1}$ Serkan Erbatur, ${ }^{1}$ \\ Nasuhi Engin Aydın, ${ }^{3}$ and Engin Burak Selcuk ${ }^{4}$ \\ ${ }^{1}$ Department of Plastic Surgery, Inonu University School of Medicine, 44380 Malatya, Turkey \\ ${ }^{2}$ Department of Plastic, Reconstructive and Aesthetic Surgery, Turgut Ozal Medical Center, Inonu University School of Medicine, \\ 44380 Malatya, Turkey \\ ${ }^{3}$ Department of Pathology, Inonu University School of Medicine, 44380 Malatya, Turkey \\ ${ }^{4}$ Department of Family Medicine, Inonu University School of Medicine, 44380 Malatya, Turkey
}

Correspondence should be addressed to Cemal Frrat, cemal.firat@inonu.edu.tr

Received 7 May 2012; Accepted 13 June 2012

Academic Editor: P. J. K. Kuppen

Copyright (C) 2012 Cemal Frrat et al. This is an open access article distributed under the Creative Commons Attribution License, which permits unrestricted use, distribution, and reproduction in any medium, provided the original work is properly cited.

\begin{abstract}
Most malignant rectal tumors are histopathologically characterized as adenocarcinoma and generally metastasize to distant organs such as the lungs or the liver. Metastasis of rectal carcinomas to the skull is extremely rare. This study reports the initial diagnosis of rectal adenocarcinoma recurrence in a 65-year-old female with scalp metastasis. The patient's history indicated a colorectal adenocarcinoma that was resected five years earlier. A skull metastasis from a rectal adenocarcinoma has not yet been reported in the literature as an initial symptom for recurrence. This paper suggests that skull metastasis from any part of the body must be considered in the differential diagnosis of soft tissue tumors in the skull even in the absence of intestinal symptoms.
\end{abstract}

\section{Introduction}

Cutaneous metastases originating from internal malignancies are uncommon. The frequency of occurrence is about $7 \%[1,2]$. Although metastatic lesions can occur at any age, their frequency tends to increase in the sixth and seventh decades of life $[3,4]$. Breast, lung, and gastrointestinal malignancies are the most likely to metastasize to the skin. However, metastases originating from gastrointestinal malignancies, such as esophageal, gastric, and colorectal tumors, are much less frequent $[5,6]$.

Skin metastases generally appear late in the course of internal malignancies, and often indicate wide-spread neoplasm. Among cutaneous metastases, the skull is a rare site compared with the nodules or alopecia neoplastica commonly detected as clinical signs. It is well-known that breast and lung carcinomas are the most common tumors that cause skull metastasis [7]. We report the unusual case of a patient with a solid nodule on the frontoparietal region of the scalp, which was diagnosed as metastatic adenocarcinoma originating from the rectum.

\section{Case Presentation}

A 65-year-old woman presented with a mass $6 \mathrm{~cm}$ in diameter located on her right frontoparietal region. The mass had appeared approximately three months earlier and had grown rapidly. The patient's history included resected colorectal adenocarcinoma followed by general surgery and was disease-free for five years. She was referred to us because the swelling and pain indicated a sebaceous cyst or a benign soft tissue tumor (Figure 1). The mass was indurated, round, and adherent to the deep planes. Mild alopecia and skin expansion due to the mass were observed on her anterior scalp. The patient complained of tenderness and mild pain when the mass was palpated. Our clinical differential diagnoses included sebaceous cyst, lipoma, or localized soft tissue tumors. But the plain radiographs and the computerized tomography demonstrated a destructive mass which was eroding the bone over the right frontoparietal region (Figure 2).

A transverse incision and a meticulous blunt dissection that were performed under local anesthesia exposed 


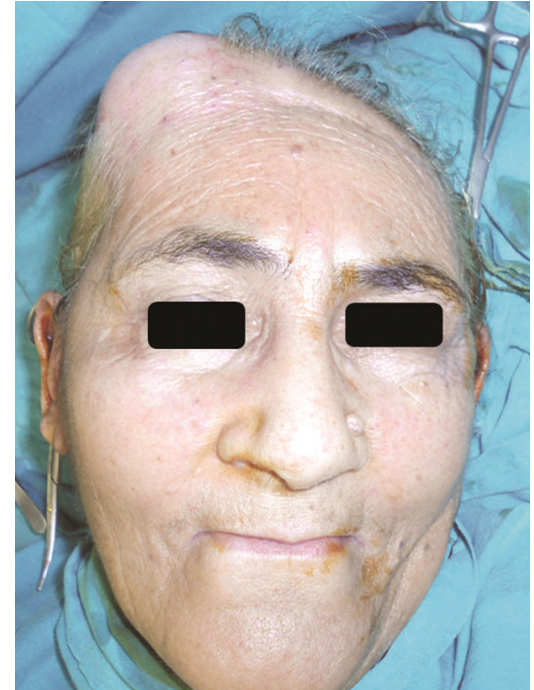

Figure 1: Preoperative view of the patient with a $6 \mathrm{~cm}$ diameter mass on the right frontoparietal region.

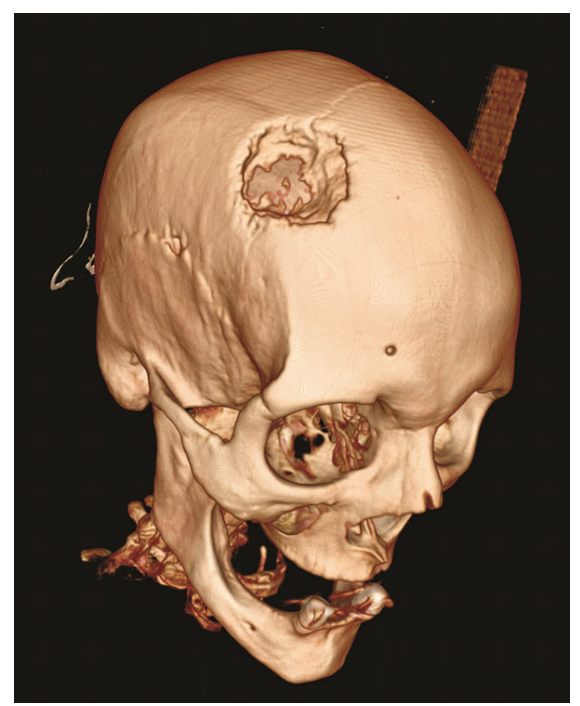

FIgURE 2: View of the mass in 3D computerized tomography.

a rubbery soft tissue mass extending to the outer table of the skull. The mass was dissected with care. However, we noticed calvarial bone destruction with an exposed duramater and lytic lesions $2 \times 3 \mathrm{~cm}$ in size around the calvarial defect. The mass was completely resected. The mass was measured to be $5.5 \times 5.0 \times 2.0 \mathrm{~cm}$ (Figure 3 ). The damaged bone sites were resected with a $1 \mathrm{~cm}$ security margin. A section of the duramater measuring $2 \times 3 \mathrm{~cm}$ was removed and the defect was reconstructed using a tensor fascia graft by the brain surgeons. Hemostasis was achieved using electrocautery and bone wax. A bolster dressing was applied following the primary closure of the skin incision. The specimen was sent for histopathological analysis. The patient was monitored closely for one day and was discharged the next day.
Histopathologic findings revealed many low differentiated adenoid, and apoptotic and necrotic spaces lined with atypical epithelial islands with vesicular nuclei. At highpower magnification, the specimens showed cytologic atypia with pleomorphic and hyperchromatic nuclei. Immunohistochemical analysis was positive for carcinoembryonic antigen (CEA), villin, and cytokeratins 7 and 20. The specimens were negative for vimentin, chromogranin, thyroglobulin, thyroid transcription factor 1 (TTF-1), and CDX2. The final diagnosis was metastatic low-differentiated adenocarcinoma compatible with the immunophenotype of colorectal adenocarcinoma (Figures 4(a), 4(b), and 4(c)). After the histopathological evaluation revealed metastatic adenocarcinoma, the patient was referred to the medical oncology department.

\section{Discussion}

Skull metastasis originating from colorectal adenocarcinomas is an unusual site. We presented here a case of metastatic rectal adenocarcinoma to the skull as an initial diagnosis of recurrence. The rectal adenocarcinoma of this patient was diagnosed and treated surgically five years earlier. The asymptomatic interval between the primary resection of the colorectal tumor and the first presentation of scalp metastasis was five years. Scalp metastasis originating from colorectal adenocarcinoma is an uncommon site. To date, only a few metastatic adenocarcinoma from primary gastrointestinal tumors have been reported to migrate to the scalp and to invade the skull $[8,9]$. These lesions usually present as firm, rubbery, and asymptomatic subcutaneous nodules. If a detailed examination and radiologic evaluation are not given, these lesions may not be differentiated from soft tissue tumors, granulomas, neurofibromas, or cysts. Histologically, the specimens were negative for CDX2 in different two analyses. Immunohistochemical analysis was positive for carcinoembryonic antigen (CEA), villin, and CK20. Villin is wellknown diagnostic marker for colorectal adenocarcinoma [10]. Another focus was not found in the investigations. These results and previous precise diagnosis were evaluated as metastatic colorectal carcinoma.

Colorectal cancer is the second most common type of primary cancer in both men and women in the United States [11]. The most frequent metastatic sites for colorectal adenocarcinoma are the liver, the peritoneum, the pelvis, the lung, and bone [12]. The average time for metastasis to appear is approximately two years after primary tumor resection $[11,12]$. Skin metastases are quite rare at the late stages of disseminated tumor, and herald a poor prognosis. However, a skin metastasis can occasionally be the initial presentation or the only sign of an asymptomatic occult neoplasm $[3,13]$. Although surgical excision cannot influence the course of the underlying disease, the mean survival time and morbidity can be ameliorated by surgery. In most cases, the abdominal wall appears to be the site preferred by skin metastases $[11,14]$.

In this case, the metastasis originating from rectal adenocarcinoma presented as a recurrence in the skull without 


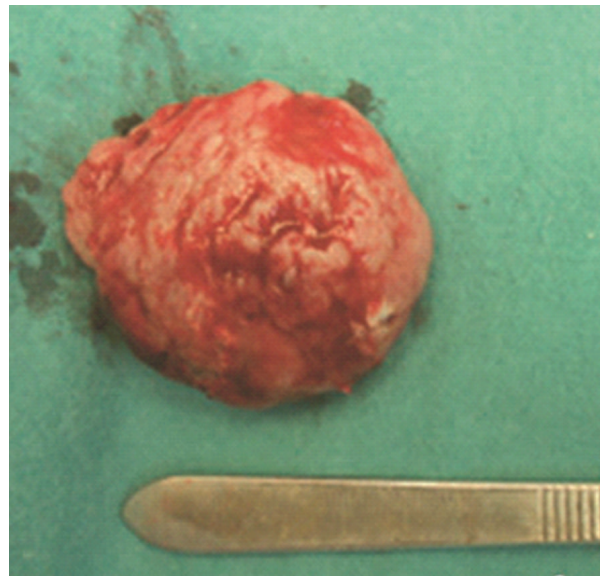

(a)

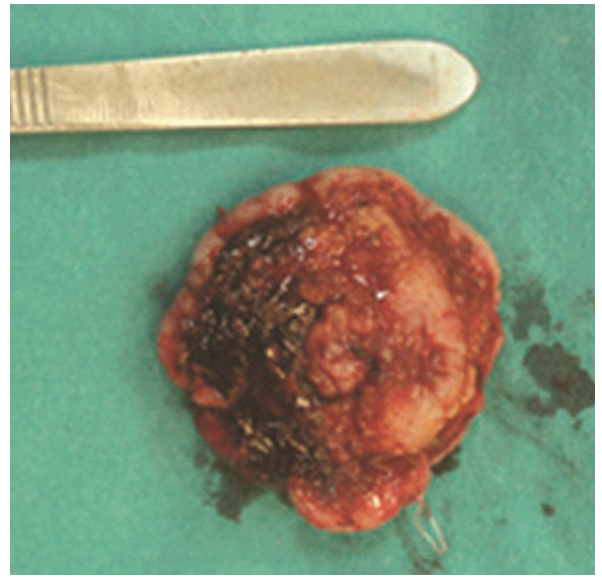

(b)

Figure 3: The macroscopic size of mass was measured $5.5 \times 5.0 \times 2.0 \mathrm{~cm}$.

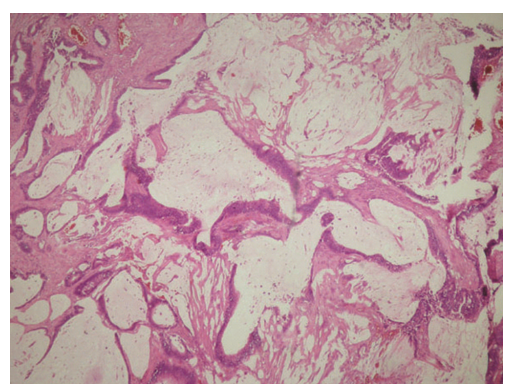

(a)

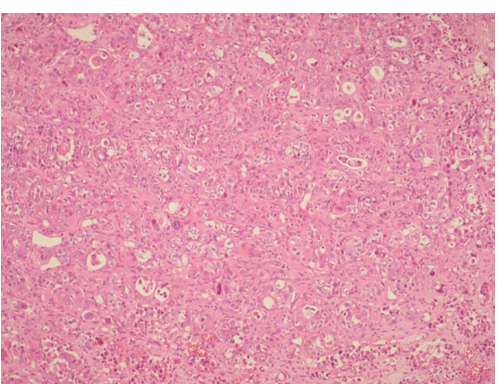

(b)

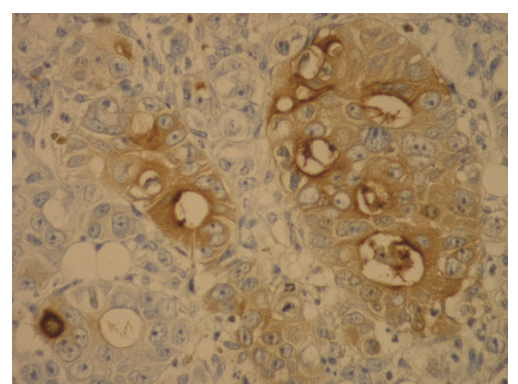

(c)

Figure 4: (a) Mucinous adenocarcinoma of the rectum, 5 years ago. (H\&E $\times 200)$, (b) low differentiated metastatic adenocarcinoma (H\&E $\times 200$ ), (c) immunohistochemical staining with monoclonal CEA positiveness (DAB chromogen $\times 400)$.

any additional metastasis in other organs such as the lung or bones, which are possible pathways for metastasis.

In conclusion, in cases where a rapidly growing mass is encountered on the scalp, metastatic adenocarcinoma originating from an internal malignancy should be taken into consideration as an initial clinical sign of rectal adenocarcinoma recurrence even in the absence of intestinal symptoms.

\section{Conflict of Interests}

The authors have no conflict of interests to disclose. Authors have no financial or proprietary interest in any instrument or product used in this study.

\section{References}

[1] P. S. Spencer and T. N. Helm, "Skin metastases in cancer patients," Cutis, vol. 39, no. 2, pp. 119-121, 1987.

[2] L. Frey, C. Vetter-Kauczok, A. Gesierich, E. B. Bröcker, and S. Ugurel, "Cutaneous metastases as the first clinical sign of metastatic gastric carcinoma," JDDG-Journal of the German Society of Dermatology, vol. 7, no. 10, pp. 893-895, 2009.
[3] J. M. Park, D. S. Kim, S. H. Oh, Y. S. Kwon, and K. H. Lee, "A case of esophageal adenocarcinoma metastasized to the scalp," Annals of Dermatology, vol. 21, no. 2, pp. 164-167, 2009.

[4] R. A. Schwartz, "Cutaneous metastatic disease," Journal of the American Academy of Dermatology, vol. 33, pp. 119-121, 1995.

[5] F. Fereidooni, K. Kovacs, M. R. Azizi, and M. Nikoo, "Skin metastasis from an occult esophageal adenocarcinoma," Canadian Journal of Gastroenterology, vol. 19, no. 11, pp. 673-676, 2005.

[6] S. Riley and T. Wah, "Cutaneous metastasis of esophageal adenocarcinoma with an unusual presentation," Journal of Clinical Ultrasound, vol. 35, no. 5, pp. 289-292, 2007.

[7] D. Yoshida, M. N. Chen, S. Awaya, and S. Nakazawa, "Cranial metastasis of hepatocellular carcinoma in a female-Case report," Neurologia Medico-Chirurgica, vol. 33, no. 12, pp. 839-844, 1993.

[8] M. V. Aydin, M. Cekinmez, O. Kizilkilic, F. Kayaselcuk, O. Sen, and N. Altinors, "Unusual case of skull metastasis secondary to pancreatic adenocarcinoma," Pathology and Oncology Research, vol. 11, no. 3, pp. 182-183, 2005.

[9] M. Igarashi, G. G. Card, P. E. Johnson, and B. R. Alford, "Bilateral sudden hearing loss and metastatic pancreatic adenocarcinoma," Archives of Otolaryngology, vol. 105, no. 4, pp. 196-199, 1979. 
[10] N. Suh, X. J. Yang, M. S. Tretiakova, P. A. Humphrey, and H. L. Wang, "Value of CDX2, villin, and $\alpha$-methylacyl coenzyme A racemase immunostains in the distinction between primary adenocarcinoma of the bladder and secondary colorectal adenocarcinoma," Modern Pathology, vol. 18, no. 9, pp. 12171222, 2005.

[11] D. Sarid, N. Wigler, Z. Gutkin, O. Merimsky, L. Leider-Trejo, and I. G. Ron, "Cutaneous and subcutaneous metastases of rectal cancer," International Journal of Clinical Oncology, vol. 9, no. 3, pp. 202-205, 2004.

[12] H. A. Hatoum, G. S. Abi Saad, Z. K. Otrock, K. A. Barada, and A. I. Shamseddine, "Metastasis of colorectal carcinoma to the testes: clinical presentation and possible pathways," International Journal of Clinical Oncology, vol. 16, pp. 203-209, 2011.

[13] S. Gómez-Diez, B. García-García, M. S. Fernández-García, and N. Pérez-Oliva, "Skin metastases as the initial presentation of pancreatic carcinoma," Actas Dermo-Sifiliograficas, vol. 101, no. 1, pp. 93-95, 2010.

[14] M. H. Brownstein and E. B. Helwig, "Patterns of cutaneous metastasis," Archives of Dermatology, vol. 105, no. 6, pp. 862$868,1972$. 


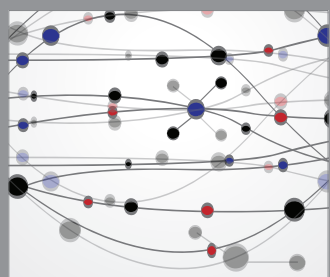

The Scientific World Journal
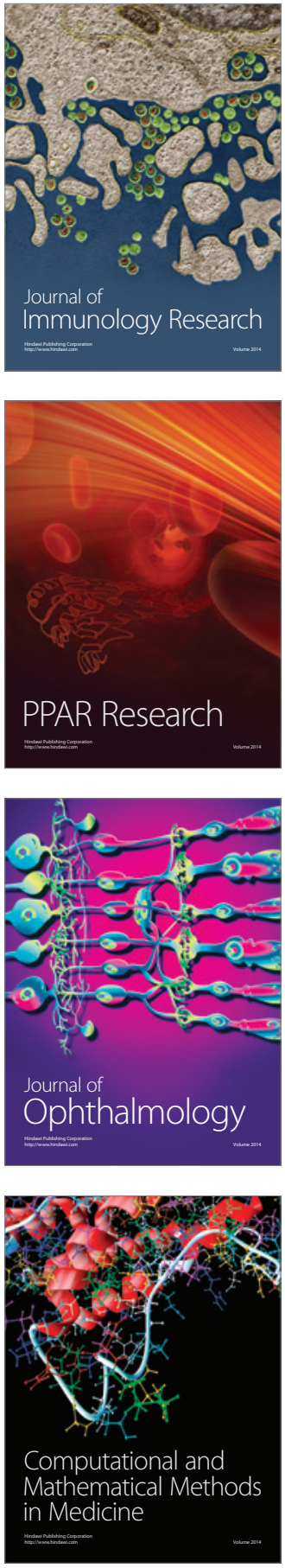

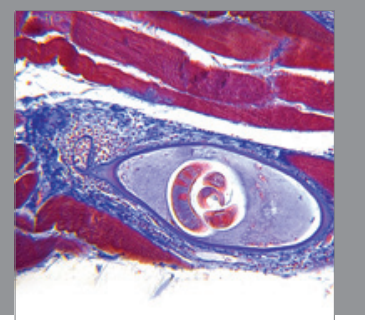

Gastroenterology

Research and Practice
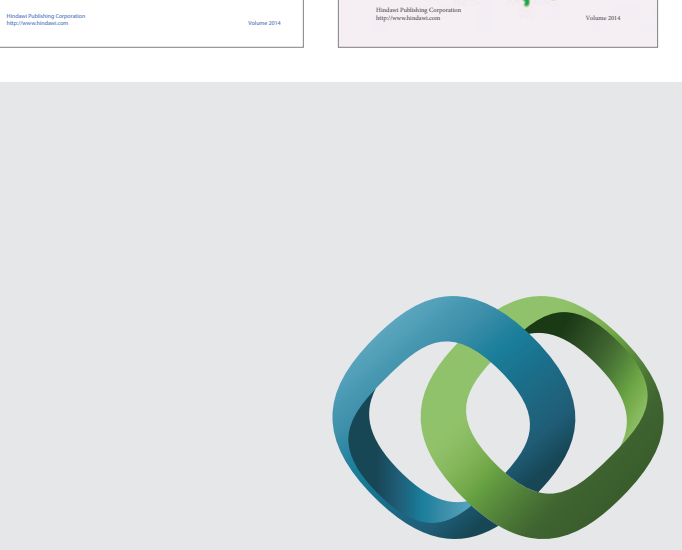

\section{Hindawi}

Submit your manuscripts at

http://www.hindawi.com
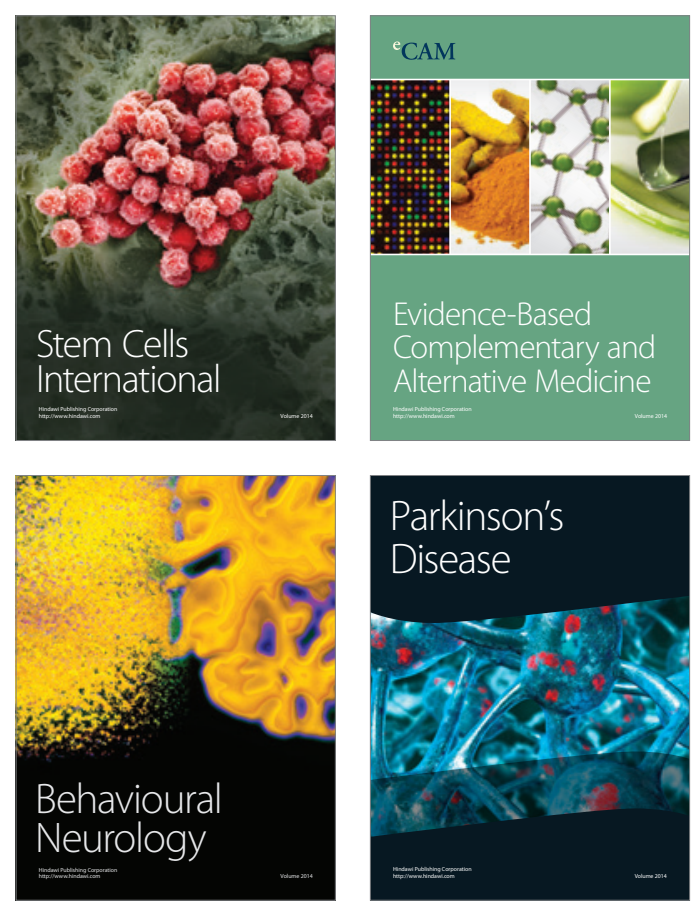

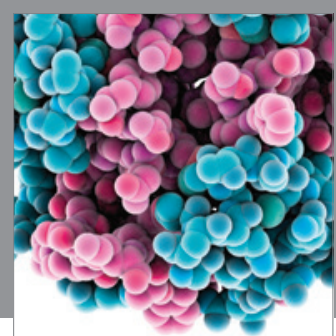

Journal of
Diabetes Research

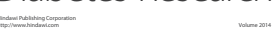

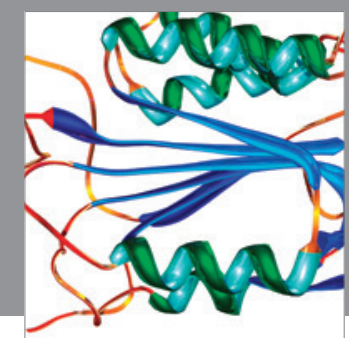

Disease Markers
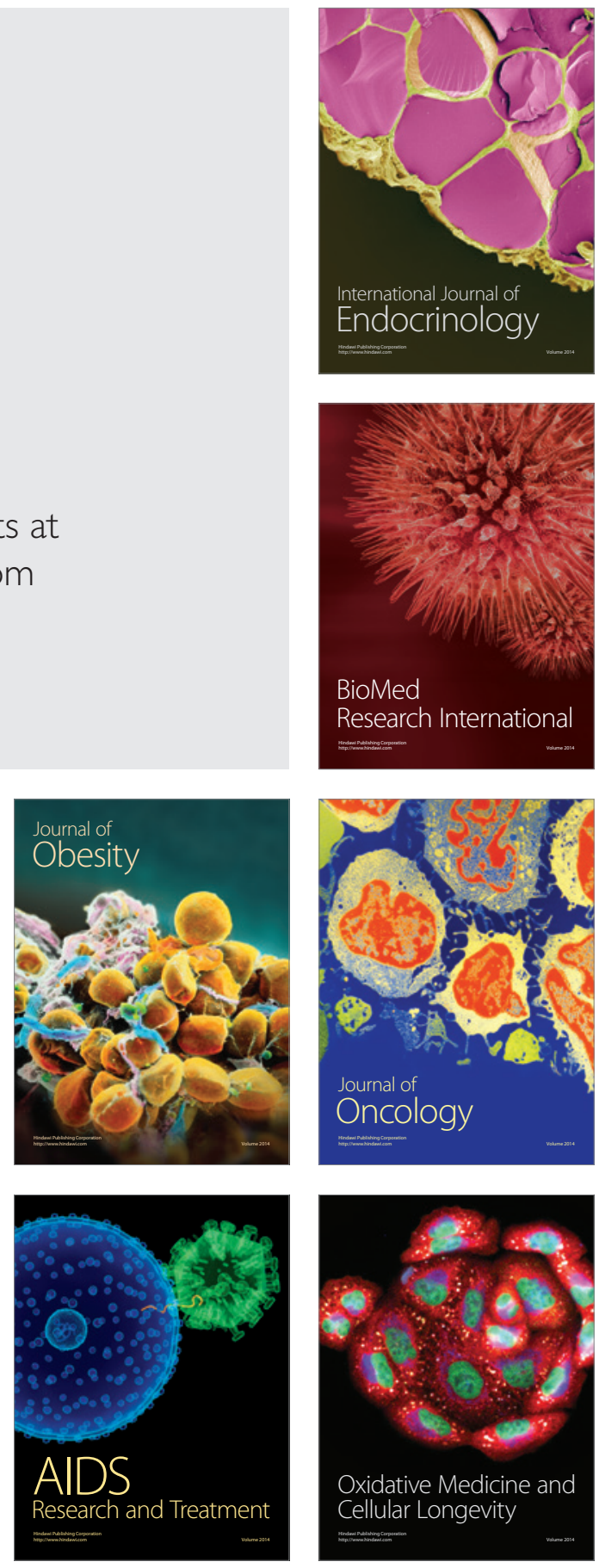